\title{
Superhydrophobic Surface Based On Assembly Of Nanoparticles For Application In Anti-icing Under Ultra-low Temperature
}

Chunhong $\mathrm{Qi}^{1}$, He Chen ${ }^{1}$, Luyan Shen ${ }^{1}$, Xiaolin $\mathrm{Li}^{1}$, Qiang $\mathrm{Fu}^{1,2}$, Yinghe Zhang ${ }^{3}$, Youyi Sun ${ }^{1 *}$, Yaqing $\operatorname{Liu}^{1 *}$

1.Shanxi Province Key Laboratory of Functional Nanocomposites, North University of China, Taiyuan 030051, P.R.China.

2. School of Civil and Environmental Engineering, University of Technology Sydney, Ultimo NSW 2007, Australia.

3.Nanotechnology Department, Helmholtz Association, Hamburg 21502, Germany.

Abstract: A new class of superhydrophobic surface based on assembly of nanoparticles were fabricated for improving mechanical durability and anti-icing performance under ultra-low temperature. Furthermore, the anti-icing performance and mechanism of the yielded superhydrophobic surface was investigated by a high speed video and thermal infrared imaging equipment. The frozen time of water droplets could be prolonged to $372.0 \mathrm{~s}$ when exposed glass slides with superhydrophobic surface to an ultra-low temperature of $-40.0^{\circ} \mathrm{C}$. This outstanding anti-icing performance is attributed to the unique structure of the superhydrophobic surface based on assembly of nanoparticles, which possesses good free-energy barrier and low heat transfer rate. This study thus opens up an avenue for the design and fabrication of superhydrophobic surface with good durability and anti-icing performance under ultra-low temperature.

Keywords: Superhydrophobic coatings, assemblies of nanoparticles, stability, anti-icing, ultra-low temperature.

Responding author: Fax: 86-351-3559669

E-mail address: syyi@pku.edu.cn (YY Sun); lyqzgz2010@163.com(YQ Liu) 


\section{Introduction}

Research on anti-icing technology has attracted increasing attention because ice adhesion and accumulation on surfaces could result in traffic interruption, communicating destruction, loss of power and damage of equipment related to aviation, telecommunication, electricity and transportation[1-2]. Icing problem was mainly solved by the active and passive deicing method[3-4]. Although the thermal melting method appears to be a straightforward anti-icing technology, it drives high energy consumption, which in turn restricts its practical applications[3]. As an alternative, superhydrophobic surface (as a passive anti-icing technique) has been considered the most promising method of anti-icing technologies owing to its advantages, such as no energy consumption, low weight and high capability for reduction of ice accumulation[5-13]. For example, the superhydrophobic surface was prepared by spaying polysiloxane-modified $\mathrm{SiO}_{2} /$ polyurethane (PU) mixing aqueous solution, which exhibited good static and dynamic anti-icing performance at $-15.0^{\circ} \mathrm{C}[4]$. The F-SiO 2 nanoparticles was dispersed in PDMS solution, which was sprayed to form superhydrophobic surface. The froze time of water droplets on its surface was extended to $276.2 \mathrm{~s}$ at $-15.0^{\circ} \mathrm{C}[11]$. The superhydrophobic surface was prepared by spaying $\mathrm{ZnO}$ dispersed in PDMS solution. It exhibited excellent anti-icing performance at $-5.0^{\circ} \mathrm{C}$ and $-10.0^{\circ} \mathrm{C}$ under inclined conditions, but the ice accretion on the superhydrophobic surface could not be avoided at $-15.0^{\circ} \mathrm{C}[12]$. The superhydrophobic surface based on aliphatic chain-substituted polybenzoxazine (PPda) and $\mathrm{SiO}_{2} \mathrm{NPs}$ was prepared by spin coating and thermal curing method. An icing delay of 386s was achieved for the water droplets on such a surface[13]. These studies demonstrate the potential of superhydrophobic surfaces for anti-icing applications. These superhydrophobic surfaces applied in anti-icing were all based on nanocomposites, in which the nanoparticles were doped into polymer matrix. In order to obtain superhydrophobicity, large surface roughness (eg. $>200 \mathrm{~nm}$ ) of these surfaces was generally needed. However, these surfaces displayed low mechanical durability due to large surface roughness, such as poor wear resistance or adhesion ability[14]. The superhydrophobicity of these surfaces was easily reduced under external forces, leading to a decrease or loss of the anti-icing performance. Therefore, there is still a great challenge to enhance the mechanical durability of superhydrophobic surface for application in anti-icing. Furthermore,,according to the classic nucleation theory, large surface roughness would promote the growth of ice nuclei under low temperature[9-10]. 
It is still a contradiction between enhancing superhydrophobicity and suppressing growth of ice nuclei for superhydrophobic surface based on nanocomposite. Therefore, the good anti-icing performance of superhydrophobic surface based on nanocomposite is difficult to be obtained under ultra-low temperature (e.g. $\left.<-30.0^{\circ} \mathrm{C}\right)$. Recently, a new class of superhydrophobic surface based on assembly of nanoparticles was proposed and prepared to improve mechanical and reproduction stability[15]. The $\mathrm{Fe}_{3} \mathrm{O}_{4} @ \mathrm{SiO}_{2}$ nanofiber nanocomposites modified with n-octyltriethoxysilane were coated on substrate by the adhension of epoxy resin. It did not only show large water contact angle (ca. $174.5^{\circ}$ ) and good mechanical durability, but also showed small surface roughness. According to previous works[15-16], the result was mainly attributed to that the $\mathrm{Fe}_{3} \mathrm{O}_{4} @ \mathrm{SiO}_{2}$ nanocomposites possessed nanofiber structure, and the single $\mathrm{SiO}_{2}$ nanoparticles without nanofiber structure showed smaller water contact angle than $150.0^{\circ}$. From above respects, although the superhydrophobic surface based on assembly of nanoparticles has been reported, yet, the synthesis process of $\mathrm{Fe}_{3} \mathrm{O}_{4} @ \mathrm{SiO}_{2}$ nanofiber nanocomposites was multi-step and complex, restricting its real application. Especially, the anti-icing performance and mechanism of superhydrophobic surface based on assembly of nanoparticles has not been reported under ultra-low temperature.

Herein, a new superhydrophobic surface based on assembly of $\mathrm{SiO}_{2}$ nanoparticles was prepared via a facile spraying method, in which the commercial $\mathrm{SiO}_{2}$ nanoparticles modified n-octyltriethoxysilane were exposed to surface and adhered on substrate by polymer. Furthermore, the preparation process was optimized to tune the structure and property of spaying surface. This superhydrophobic surface did not only show excellent superhydrophobicity, but also showed low surface roughness under optimum condition. Such superhydrophobic surfaces can effectively delay the formation and growth of ice nuclei and exhibit good mechanical durability due to their low surface roughness. Therefore, this novel superhydrophobic surface based on assembly of nanoparticles is expected to apply in practical anti-icing technology under ultra-low temperature.

2. Experimental

\subsection{Materials}

Ethanol $(\mathrm{EtOH})$, toluene and $\mathrm{SiO}_{2}$ nanoparticles were purchased from Tianjin Guangfu Technology Development Co., Ltd. Epoxy resin and curing agent was purchased from Shanghai Yue Ke Composite Co., Ltd. N-octyltriethoxysilane was 
purchased from Aladdin. Q235 and transparent glass slides were purchased from local chemical market. All reagents were used without further purification.

2.2 Preparation of superhydrophobic surface based on assembly of $\mathrm{SiO}_{2}$ nanoparticles via spray-coating

The superhydrophobic surface based on assembly of nanoparticles was prepared by a two-step approach as shown in following. Firstly, the $\mathrm{SiO}_{2}$ nanoparticles with low surface energy were synthesized. $5.0 \mathrm{~g} \mathrm{SiO}_{2}$ nanoparticles were dispersed in $100.0 \mathrm{~mL}$ toluene under a mechanical stirring for 1.0 hour, followed by addition of $10.0 \mathrm{~mL}$ n-octyltriethoxysilane (OTS). The reaction mixture was then heated to $120.0^{\circ} \mathrm{C}$ for 12.0 hours to afford OTS modified $\mathrm{SiO}_{2}$ nanoparticles $\left(\mathrm{OTS}-\mathrm{SiO}_{2}\right)$. Thereafter, the OTS-SiO${ }_{2}$ nanoparticles were dispersed in ethanol to form a homogeneous OTS-SiO ${ }_{2}$ dispersion solution with different concentrations of $1.0 \mathrm{wt} \%$, $2.0 \mathrm{wt} \%, 5.0 \mathrm{wt} \%$ and $10.0 \mathrm{wt} \%$. Secondly, the epoxy resin and amine curing agent $(\mathrm{m} / \mathrm{m}=4: 1)$ were mixed to form a paint. The above resin paint was sprayed onto the substrates and pre-cured at $80.0^{\circ} \mathrm{C}$. Then the $\mathrm{OTS}-\mathrm{SiO}_{2}$ dispersion solution was sprayed onto epoxy resin coating. To achieve a uniform coating, the spray airbrush was placed perpendicular to the substrates at the optimized distance of $15.0 \mathrm{~cm}$ and moved from top to bottom at a constant speed. The resultant composite coatings were further completely cured at room temperature for 2.0 hours.

\subsection{Instruments and characterization}

The surface morphology of materials was determined by field emission scanning electron microscopy (SEM, Su-8010) and transmission electron microscopy (TEM, JEM-2010).

Power X-ray diffraction patterns of $\mathrm{SiO}_{2}$ and modified $\mathrm{SiO}_{2}\left(\mathrm{OTS}_{-} \mathrm{SiO}_{2}\right)$ nanoparticles were obtained using a Smartlab-3 (Rigaku, Japan). Each pattern was collected over the $2 \theta$ range of $10.0-80.0^{\circ}$ with a scanning speed of $4.0^{\circ} / \mathrm{min}$ at $35.0 \mathrm{kV}$ and $40.0 \mathrm{~mA}$.

Fourier transform infrared spectrometer (FT-IR) (Thermo Nicolet 360) was conducted using a Thermo Nicolet 360 .

The $3 \mathrm{D}$ surface topography of the coating was measured using a non-contact white light profilometer.

The contact angles, sliding angles and supplementary videos were recorded using a DSA100 contact angle tester (Germany). Here, water droplets $(10.0 \mu \mathrm{L})$ were carefully dropped onto the substrates at least five different locations. 
The pull-off tests were conducted by GB/T 5210-2006 to measure the adhesion strength of the coating onto the substrates.

\subsection{Anti-icing tests}

The resultant surface was placed at an angle of approximately $10.0^{\circ}$ and cooled down at $-20.0^{\circ} \mathrm{C}$ to $-40.0^{\circ} \mathrm{C}$ for $30.0 \mathrm{~min}$. Then, the water droplets were dropped on the surface, where the icing process and temperature of water droplets were recorded using a high speed video and thermal infrared imaging equipment, respectively.

\subsection{Mechanical durability}

Sandpaper abrasion tests were carried out to examine the robustness of the anti-icing coating. The coated surface was placed face down to the sandpaper (Silicon carbide sandpaper, Grit No. 240), and longitudinally and transversely $(10.0 \mathrm{~cm}$ for each direction) abraded by the sandpaper under a weight of $50.0 \mathrm{~g}$. This process was defined as one cycle. The contact angle of the coated surface was then measured as a function of abrasion cycles. Anti-icing performance of the coated surface was evaluated again after 50 abrasion cycles.

\section{Results and Discussion}

In this study, novel OTS-SiO 2 nanoparticles were prepared and dispersed in ethanol. This mixture was then spray-coated on an epoxy resin-based pre-layer to afford a superhydrophobic surface with nanoengineered structure based on assembly of $\mathrm{SiO}_{2}$ nanoparticles. Fig.1A shows the XRD patterns of pristine $\mathrm{SiO}_{2}$ and $\mathrm{OTS}-\mathrm{SiO}_{2}$ nanoparticles. A broad peak at $23.0^{\circ}$ was observed in both patterns, which was assigned to the amorphous state of $\mathrm{SiO}_{2}[14]$. The chemical structures of pure $\mathrm{SiO}_{2}$ and OTS-SiO 2 nanoparticles were further analyzed by IR spectra (Fig.1B). A strong absorption peak at $1105.0 \mathrm{~cm}^{-1}$ was observed for both samples, which was assigned to Si-O-Si stretching vibration of $\mathrm{SiO}_{2}$ [17]. The absorption peaks at $3440.0 \mathrm{~cm}^{-1}$ and $1646.0 \mathrm{~cm}^{-1}$ were assigned to $\mathrm{O}-\mathrm{H}$ stretching vibrations of $\mathrm{SiO}_{2}$ [18]. After surface modification of OTS, the absorption peak at $1646.0 \mathrm{~cm}^{-1}$ disappeared. Meanwhile, new absorption peaks at $2850.0 \mathrm{~cm}^{-1}$ and $2923.0 \mathrm{~cm}^{-1}$ were observed, which were assigned to the $-\mathrm{CH}_{2}$ - and $-\mathrm{CH}_{3}$ stretching vibrations of OTS, respectively[15, 17]. These results confirmed that the OTS was chemically grafted on the surface of $\mathrm{SiO}_{2}$ nanoparticles. The micro-structure of OTS-SiO 2 nanoparticles was further characterized by the SEM image. It clearly showed spherical nanoparticles with a diameter of $c a$. $25.0 \mathrm{~nm}$ (Fig.1C). This result was further confirmed by TEM measurements as shown in Fig.1D. The energy-dispersive X-ray spectrum (EDS) of $\mathrm{OTS}_{-} \mathrm{SiO}_{2}$ nanoparticles was 
also characterized as shown in Fig.1E. It was clearly observed the $\mathrm{Si}, \mathrm{C}$ and $\mathrm{O}$ elements. According to the weight ratio of each element, the calculated weight content of OTS was of $11.6 \%$ of OTS-SiO 2 nanoparticles. Of particular note, we didn't observed any peaks corresponding to other elements. These results therefore confirmed the successful preparation of $\mathrm{OTS}-\mathrm{SiO}_{2}$ nanoparticles with high purity.

\section{Fig.1.}

The engineered nanostructure of the superhydrophobic surface based on assembly of OTS-SiO 2 nanoparticles was characterized by SEM images as shown in Fig.2. As shown in Fig.2A, the pure epoxy resin (EP) coating clearly exhibited a smooth surface. After spray-coating of OTS-SiO${ }_{2}$ nanoparticles, the surface became rougher due to the assembly of OTS-SiO 2 nanoparticles (Fig.2B-C and Figure S1). The cross-section structure of $\mathrm{OTS}-\mathrm{SiO}_{2} / \mathrm{EP}$ was further characterized and compared by the optical microscope as shown in Fig.2D-E. A double-layer structure was clearly observed, in which the OTS-SiO 2 nanoparticles were adhesive on surface of EP. The thickness of topcoat based on assembly of $\mathrm{OTS}-\mathrm{SiO}_{2}$ nanoparticles was about $2.5 \mu \mathrm{m}$. The roughness of the resultant surface was further characterized by three-dimensional stereoscopic diagram as shown in Fig.2F-G. After spray-coating of $\mathrm{OTS}^{-\mathrm{SiO}_{2}}$ nanoparticle dispersion solution with $2.0 \mathrm{wt} \%$, the Sq of surface was only $c a .0 .099 \mu \mathrm{m}$. However, it exhibited a high WAC of $c a .150 .7^{\circ}$ (inset picture in Fig.2F), indicating a superhydrophobicity. When the concentration of $\mathrm{OTS}-\mathrm{SiO}_{2}$ dispersion solution was improved to $5.0 \mathrm{wt} \%$, the $\mathrm{Sq}$ of surface based on assembly of $\mathrm{OTS}_{\mathrm{SiO}}$ nanoparticles significantly increased to $0.254 \mu \mathrm{m}$. As expected, the surface with higher Sq value led to a higher WCA of $173.1^{\circ}$ (inset picture in Fig.2G), indicating a better superhydrophobicity. In a comparison, when the concentration of $\mathrm{OTS}-\mathrm{SiO}_{2}$ dispersion solution was decreased to about $1.0 \mathrm{wt} \%$, the surface based on assembly of OTS-SiO${ }_{2}$ nanoparticles showed a low WAC of about $139.6^{\circ}$, indicating a hydrophobicity ( Figure S1B). Although the concentration of OTS-SiO 2 dispersion solution was further improved to $10.0 \mathrm{wt} \%$, yet the WCA (ca. $172.9^{\circ}$ ) of surface was few change comparing to surface prepared by $5.0 \mathrm{wt} \%$ dispersion solution ( Figure S1A). These results confirmed the successful fabrication of superhydrophobic coatings based on assembly of OTS-SiO 2 nanoparticles, where the WCA could be easily tuned by varying the nanoparticle' concentration range of $1.0 \mathrm{wt} \% \sim 5.0 \mathrm{wt} \%$.

Fig.2. 
The superhydrophobic surface was usually easily destroyed by mechanical forces due to poor adhensive ability of superhydrophobic surface on substrate. So, the adhensive ability of superhydrophobic surface based on assembly of OTS-SiO nanoparticles was detected and evaluated by pull-out tests as shown in Fig.3. Fig.3A shows the optical photographs of various surfaces after pull-out test. The adhesion strength of pristine epoxy resin coating was about 5.6MPa as shown in Fig.3B. After the covering OTS-SiO 2 nanoparticles, the adhesion strength was obviously improved to $7.8 \mathrm{MPa}$. The good adhesion strength of superhydrophobic surface based on nanocomposite was difficult to be observed in previous works[5-11], in which the particles were doped into polymer with high concentration, decreasing the adhensive property of polymer matrix. Here, the enhanced adhesion strength of surface based on assembly of OTS-SiO 2 nanoparticles was attributed to that the $\mathrm{OTS}-\mathrm{SiO}_{2}$ nanoparticles slightly affected the adhensive property of EP on substrate, contrarily could improve mechanical strength of EP coating. Whatever the adhesion strength of surface on substrate was mainly attributed to adhensive property of polymer matrix. At the same above reason, it showed a similar adhesion strength of surface based on assembly of OTS-SiO ${ }_{2}$ nanoparticles prepared by various concentrations of $\mathrm{OTS}-\mathrm{SiO}_{2}$ nanoparticle dispersion solution. These result indicated the good adhesion strength of present superhydrophobic surface based on assembly of nanoparticles, which was also key role for improving mechanical durability of anti-icing surface.

\section{Fig.3.}

Sliding angle (SA) of the resultant superhyhydrophobic surface based on assembly of OTS-SiO 2 nanoparticles was then characterized and compared as shown in Fig.4. It displayed the approach, contact, bounce and departure of the water droplet from the surface in the bouncing tests for both samples. For the superhyhydrophobic surface with a WCA of $173.1^{\circ}$ (Fig.4A), it took $264.0 \mathrm{~ms}$ for the water droplet rolling off surface with an slope angle of $c a .0^{\circ}$ (in Video S1), suggesting an low SA of $c a .0^{\circ}$ for aforementioned superhyhydrophobic surface. In contrast, the superhyhydrophobic surface with a WCA of $150.7^{\circ}$ displayed an higher slope angle of $c a .5^{\circ}$ (Fig.4B) (in Video S2). It therefore could be concluded that the SA decreased with increasing in WCA of superhyhydrophobic surface based on assembly of OTS-SiO ${ }_{2}$ nanoparticles. This result was attributed to the reduced direct contact area between water and superhydrophobic surface with a higher WCA[19], resulting in a faster rolling off rate of water droplets from such surface[20]. This small SA of novel superhyhydrophobic 
surface based on assembly of OTS-SiO 2 nanoparticles was very important to improve anti-icing performance.

\section{Fig.4.}

The anti-icing ability of superhyhydrophobic surface based on assembly of nanoparticles was then evaluated using a high speed video equipment as shown in Fig.5. When the falling droplet first encountered the superhyhydrophobic surface with WCA of $173.1^{\circ}$ from a height of $10.0 \mathrm{~cm}$, it broadened, then bounced, and after a few cycles, finally rolled off the surface with an slope angle of $0^{\circ}$ at different cold temperatures of $-20.0^{\circ} \mathrm{C}$ and $-30.0^{\circ} \mathrm{C}$ as shown in Fig.5A-B, respectively. When further reducing temperature to $-40.0^{\circ} \mathrm{C}$, the water droplets could not roll off but adhered on the superhyhydrophobic surface at an slope angle of $0^{\circ}$ (Fig.5C). Only when the surface was placed at an higher slope angle of approximately $10^{\circ}$, the water droplet could rapidly roll off surface at $-40.0^{\circ} \mathrm{C}$ (Fig.5D). Nevertheless, the presented superhydrophobic coating displayed an low slope angle at $-40.0^{\circ} \mathrm{C}$, which was rarely reported in previous works[5-9, 21]. In another case, the water droplets could not roll off and adhered on the superhydrophobic coating with a WCA of $150.7^{\circ}$ and an slope angle of $10^{\circ}$ or $0^{\circ}$ at $-20.0^{\circ} \mathrm{C}$ (Fig.5E-F). These results indicated that the superhyhydrophobic surface based on assembly of nanoparticles with a WCA of $173.1^{\circ}$ displayed a strong anti-icing ability under ultra-low temperature of $-40.0^{\circ} \mathrm{C}$.

\section{Fig.5.}

The icing process of water droplets on the superhyhydrophobic surface based on assembly of nanoparticles was further monitered by the high speed video equipment as showed in Fig.6. When the water drop began to freeze, the transparent center of the condensed water droplet vanished due to the difference in reflectivity between water and ice crystal[22]. We observed that ice crystalline grew from bottom to the top after a cooling period for water droplets on superhyhydrophobic surface. For the superhyhydrophobic surface with a WCA of $173.1^{\circ}$, the water droplets took 520.0s and 372.0 s to completely freeze at $-30.0^{\circ} \mathrm{C}$ and $-40.0^{\circ} \mathrm{C}$, respectively (Fig.6A-B). For the superhyhydrophobic surface prepared by $10.0 \mathrm{wt} \%$ nanoparticle dispersion solution, the water droplets took similar time (ca.380.0s) to completely freeze at $-40.0^{\circ} \mathrm{C}$ due to similar WCA of $172.9^{\circ}$ as shown in Figure S2A. In contrast, it took 262.0s and 215.0s for water droplets to freeze completely on the superhyhydrophobic surface with a WCA of $150.7^{\circ}$ at $-30.0^{\circ} \mathrm{C}$ and $-40.0^{\circ} \mathrm{C}$, respectively (Fig.6C-D). However, for the surface prepared by prepared by $1.0 \mathrm{wt} \%$ nanoparticle dispersion solution, the water droplets 
only took 149.0 s completely freeze at $-40.0^{\circ} \mathrm{C}$ as show in Figure S2B. These results thus suggested that the presented superhydrophobic surface based on assembly of nanoparticles could effectively prolong froze time of water droplet, in which the froze time on surface based on assembly of nanoparticles increased with increasing in WCA. In addition, these results therefore also revealed that the good anti-icing ability of superhyhydrophobic surface based on assembly of nanoparticles was attributed to prolong froze time, strongly depending on the WCA and sliding angle.

\section{Fig.6.}

The temperature of water droplets on superhyhydrophobic surface based on assembly of nanoparticles was also characterized by thermal infrared imaging equipment as shown in Fig.7. Fig.7A-D showed the thermal infrared images of water droplets on the superhyhydrophobic surface as a function of cooling time at $-30.0^{\circ} \mathrm{C}$ and $-40.0^{\circ} \mathrm{C}$. The transient temperature profiles of the water droplets were further summarized in Fig.7E. When the superhyhydrophobic surface was remained under ultra-low temperature (e.g. $-30.0^{\circ} \mathrm{C}$ and $-40.0^{\circ} \mathrm{C}$ ), all the curves (temperature $v s$. time) displayed similar trend. The temperature of water droplets gradually decreased with increasing in cooling time. It took $180.0 \mathrm{~s}$ and $90.0 \mathrm{~s}$ for the water droplet to cool down ca. $0.6^{\circ} \mathrm{C}$ at $-30.0^{\circ} \mathrm{C}$ on superhyhydrophobic surface with different WCAs of $173.1^{\circ}$ and $150.7^{\circ}$, respectively. These results suggested that the presented superhyhydrophobic surface based on assembly of nanoparticles could effectively reduce the heat transfer rate between surface and water droplet, decreasing the cooling rate of water droplet. In addition, a dramatical decrease in temperature was observed for all curves, corresponding to the formation of ice nucleation. We observed that it took $180.0 \mathrm{~s}$ and $120.0 \mathrm{~s}$ to form ice nucleation (an important indicator of water freezing) for superhyhydrophobic surfaces with the WCA of $173.1^{\circ}$ and $150.7^{\circ}$, respectively. These results thus suggested that the good anti-ability of present superhyhydrophobic surface based on assembly of nanoparticles was also attributed to its low heat transfer rate, prolonging the formed time of ice nucleation.

\section{Fig.7.}

We further compared the anti-icing performance of present superhydrophobic surface with other anti-icing surfaces as shown in Table 1. It was concluded that present surface based on assembly of nanoparticles exhibited longer froze time as well as good anti-icing performance under ultra-low temperature, i.e. $-40.0^{\circ} \mathrm{C}$. The good anti-icing performance was mainly attributed to the assembly of $\mathrm{OTS}_{-} \mathrm{SiO}_{2}$ 
nanoparticles with low surface energy adhension on substrate by EP. Firstly, the OTS-SiO ${ }_{2}$ nanoparticles exposed to surface provided excellent superhydrophobicity $\left(\mathrm{CA} \sim 173.1^{\circ}\right)$ due to low surface energy and roughness. According to Cassie-Baxter model[23-24]

$$
\cos \theta_{c}=f\left(\cos \theta_{0}+1\right)-1
$$

where $\theta_{c}$ represents the apparent contact angle, $\theta_{0}$ represents the intrinsic contact angle and $f$ represents the fraction of solid-liquid contact area. It was found that when the WAC of superhydrophobic surface was improved from $150.7^{\circ}$ to $173.1^{\circ}$, the solid-liquid contact area fraction increased from $13.5 \%$ to $1.9 \%$. So, the excellent superhydrophobicity (or large WCA) could reduce contact area between water droplets and surfaces as shown in Scheme 1A. At the same time, the air film was easily formed on superhydrophobic surface with excellent superhydrophobicity, decreasing the heat transfer rate between surface and water droplets. The decrease of contact area and heat transfer rate between surface and water droplets prolonged the cooling time for droplets to reach nucleation temperature. Secondly, the OTS-SiO 2 nanoparticles exposed to surface provided super small SA $\left(c a .0^{\circ}\right)$ due to low surface energy and small roughness. The super small SA was beneficial to that the water droplets rapidly rolled off surface before it froze. Thirdly, present superhydrophobic surface showed good free-energy barrier for formation of ice nucleation, resulting from small size of surface based on assemblies of nanoparticles as shown in Scheme 1A. As well-known, the size of surface roughness was smaller, and the ice nucleation was more difficult to form[9]. At the same reason, although the superhydrophobic surface based on nanocomposite coating showed good superhydrophobicity (in Scheme 1B), yet it still showed relatively poor anti-icing performance in previous works due to be large surface roughness[25-29]. Here, the formation of air film was characterized and confirmed by the optical photograph as shown in Scheme 1C. When the superhydrophobic surface was inserted into water, the surface tension and capillary force of water could form depressions similar to meniscus on the surface of the coating (in Scheme 1C), which was macroscopically represented as an air film[15, 30]. In addition, it was found that the thickness of air film increased with increasing in superhydrophobicity. Based on above discussions, the anti-icing ability of surface was not only closely correlated to the WAC and SA, but also determined by the nano-structure of the surface.

\section{Scheme 1.}




\section{Table 1.}

It is well-known that the mechanical durability of superhydrophobic surface is generally poor, resulting in poor practicality of anti-icing coatings. Here, the mechanical durability of the presented anti-icing coating was further evaluated by sandpaper abrasion tests as shown in Fig.8A. It was found that the water droplets could rapidly roll off the surface after 50 abrasion cycles. Fig. $8 \mathrm{~B}$ shows the WCA and SA of superhydrophobic surface based on assembly of $\mathrm{OTS}-\mathrm{SiO}_{2}$ nanoparticles as a function of abrasion cycles. The WCA values maintained consistent around $165.5^{\circ}$ and SA values were still below $10^{\circ}$ with increasing in abrasion cycles. Although the abraded surfaces would show peaks and valleys in the longitudinal and transverse grinding directions (in Fig.8C), yet it showed similar WAC and hydrophobicity for various area of surface. In additon, the surface roughness of superhydrophobic surface based on assembly of OTS-SiO 2 nanoparticles was slight change before and after 50 abrasion cycles by comparing Fig.2G with Fig.8D. These results suggested excellent mechanical durability of superhydrophobic surface based on assembly of OTS-SiO nanoparticles for application in anti-icing. The good mechanical stability was attributed to the unique structural characters of OTS-SiO ${ }_{2}$ nanoparticles exposed to surface and adhered on substrate by EP, resulting in low surface roughness ( $c a$. $0.254 \mu \mathrm{m}$ ) (in Fig.2), strong adhesion between nanoparticles and the substrate (Fig.3) and good wear resistance of inorganic $\mathrm{SiO}_{2}$ nanoparticles. As expected, the water droplets could also rapidly roll off the surface at an slope angle of approximately $10^{\circ}$ after 50 abrasion cycles at ultra-low temperature, i.e. $-40.0^{\circ} \mathrm{C}$ (Fig. $\left.8 \mathrm{E}\right)$. These results confirmed the good mechanical stability of superhydrophobic surface based on assembly of OTS-SiO 2 nanoparticles for application in anti-icing under ultra-low temperature.

\section{Fig.8.}

\section{Conclusions}

A new class of superhydrophobic surface based on assembly of nanoparticles was fabricated for application in anti-icing under ultra-low temperature. The unique nanostructure of the presented surfuce provided excellent superhydrophobicity, super small sliding angle, and prolonged the formation time of ice nucleation, resulting in good anti-icing ability under ultra-low temperature. Furthermore, the nanoparticles with low surface energy was exposed to surface and adhered on substrate by the polymer, exhibiting good mechanical durability. These results presented in this work 
will provide insights into this complex phenomenon and open up new avenues for the rational design of anti-icing, superhydrophobic surfaces by tuning surface nanostructure.

Acknowledgments

The authors are grateful for the support of the National Natural Science Foundation of China under grants (51773184 and U1810114), Shanxi Provincial Natural Science Foundation of China (201701D121046 and 201803D421081), and the ARC grants under the Future Fellowship scheme (FT180100312).

Supporting Information

Support: Structure and property of surface prepared by nanoaprticle dispersion solution with various concentration of $1.0 \mathrm{wt} \%$ and $10.0 \mathrm{wt} \%$.

Video S1: Water droplet rolling on superhydrophobic surface with contact angle of $173.1^{\circ}$.

Video S2: Water droplet rolling on superhydrophobic surface with contact angle of $150.7^{\circ}$.

Reference

[1] Hao, P.; Lv, C.; Zhang, X. Freezing of sessile water droplets on surfaces with various roughness and wettability, Applied Physics Letters, 104 (2014) 161609.

[2] Dou, R.; Chen, J.; Zhang, Y.; Wang, X.; Cui, D.; Song, Y.; Jiang, L.; Wang, J. Anti-icing coating with an aqueous lubricating layer, ACS applied materials \& interfaces, 6 (2014) 6998-7003.

[3] Zhu, C.; Liu, S.; Shen, Y.; Tao, J.; Wang, G.; Pan, L. Verifying the deicing capacity of superhydrophobic anti-icing surfaces based on wind and thermal fields, Surface and Coatings Technology, 309 (2017) 703-708.

[4] Li, Y.; Li, B.; Zhao,X.; Tian, N.; Zhang, J. Totally Waterborne, Nonfluorinated, Mechanically Robust, and Self-Healing Superhydrophobic Coatings for Actual Anti-Icing, ACS applied materials \& interfaces, 10 (2018) 39391-39399.

[5] Antonini, C.; Innocenti, M.; Horn, T.; Marengo, M.; Amirfazli, A. Understanding the effect of superhydrophobic coatings on energy reduction in anti-icing systems, Cold Regions Science and Technology, 67 (2011) 58-67.

[6] Fu, S. P.; Sahu, R. P.; Diaz, E.; Robles, J. R.; Chen, C.; Rui, X.; Klie, R. F.; Yarin, A. L.; Abiade, J. T. Dynamic Study of Liquid Drop Impact on Supercooled Cerium 
Dioxide: Anti-Icing Behavior, Langmuir : the ACS journal of surfaces and colloids, 32 (2016) 6148-6162.

[7] Shen, Y.; Wang, G.; Tao, J.; Zhu, C.; Liu, S.; Jin, M.; Xie, Y.; Chen, Z. Anti-Icing Performance of Superhydrophobic Texture Surfaces Depending on Reference Environments, Advanced Materials Interfaces, 4 (2017) 1700836.

[8] Yilbas, B. S.; Hassan, G.; Al-Sharafi, A.; Ali, H.; Al-Aqeeli, N.; Al-Sarkhi, A. Water Droplet Dynamics on a Hydrophobic Surface in Relation to the Self-Cleaning of Environmental Dust, Scientific reports, 8 (2018) 2984.

[9] Eberle, P.; Tiwari, M. K.; Maitra, T.; Poulikakos, D. Rational nanostructuring of surfaces for extraordinary icephobicity, Nanoscale, 6 (2014) 4874-4881.

[10] Fu, Q.; Wu, X.; Kumar, D.; Ho, J. W.; Kanhere, P. D.; Srikanth, N.; Liu, E.; Wilson, P.; Chen, Z. Development of sol-gel icephobic coatings: effect of surface roughness and surface energy, ACS applied materials \& interfaces, 6 (2014) 20685-20692.

[11] Shen, Y.; Wu, Y.; Tao, J.; Zhu, C.; Chen, H.; Wu, Z.; Xie, Y. Spraying Fabrication of Durable and Transparent Coatings for Anti-Icing Application: Dynamic Water Repellency, Icing Delay, and Ice Adhesion, ACS applied materials \& interfaces, 11 (2019) 3590-3598.

[12] Yang, C.; Wang, F.; Li, W.; Ou, J.; Li, C.; Amirfazli, A. Anti-icing properties of superhydrophobic ZnO/PDMS composite coating, Applied Physics A, 122 (2015)1-10. [13]Zhang, H.; Lu, X.; Xin, Z.; Zhang, W.; Zhou, C. Preparation of superhydrophobic polybenzoxazine/ $\mathrm{SiO}_{2}$ films with self-cleaning and ice delay properties, Progress in Organic Coatings, 123 (2018) 254-260.

[14] Guo, Q.; Huang, D.; Kou, X.; Cao, W.; Li, L.; Ge, L.; Li, J. Synthesis of disperse amorphous $\mathrm{SiO}_{2}$ nanoparticles via sol-gel process, Ceramics International, 43 (2017) 192-196.

[15]Zhou, Y.; Ma, Y.; Sun, Y.; Xiong, Z.; Qi, C.; Zhang, Y.; Liu, Y. Robust superhydrophobic surface based on multiple hybrid coatings for application in corrosion protection, ACS Appl Mater Interfaces, 11(2019)6512-6526

[16] Alamri, H.; Shahrani, A. A.; Bovero, E.; Khaldi, T.; Alabedi, G.; Obaid, W.; Taie, I. A.; Fihri, A. Self-cleaning superhydrophobic epoxy coating based on fibrous 
silica-coated iron oxide magnetic nanoparticles, Journal of Colloid and Interface Science, 513(2018)349-356.

[17]Cui, M.; Shen, Y.; Tian, H.; Yang, Y.; Feng, H.; Li, J. Influence of water adhesion of superhydrophobic surfaces on their anti-corrosive behavior, Surface and Coatings Technology, 347 (2018) 38-45.

[18] Sun, H.; Xu, Y.; Zhou, Y.; Gao, W.; Zhao, H.; Wang, W. Preparation of superhydrophobic nanocomposite fiber membranes by electrospinning poly(vinylidene fluoride)/silane coupling agent modified $\mathrm{SiO}_{2}$ nanoparticles, Journal of Applied Polymer Science, 134 (2017)44501.

[19] Zhou, H.; Chen, R.; Liu, Q.; Liu, J.; Yu, J.; Wang, C.; Zhang, M.; Liu, P.; Wang, J. Fabrication of $\mathrm{ZnO}$ /epoxy resin superhydrophobic coating on AZ31 magnesium alloy, Chemical Engineering Journal, 368 (2019) 261-272.

[20] Zhang, Z. H.; Wang, H. J.; Liang, Y. H.; Li, X. J.; Ren, L. Q.; Cui, Z. Q.; Luo, C. One-step fabrication of robust superhydrophobic and superoleophilic surfaces with self-cleaning and oil/water separation function, Scientific reports, 8 (2018) 3869 .

[21] Qing, Y.; Long, C.; An, K.; Hu, C.; Liu, C. Sandpaper as template for a robust superhydrophobic surface with self-cleaning and anti-snow/icing performances, J Colloid Interface Sci, 548 (2019) 224-232.

[22] Zhan, X.; Yan, Y.; Zhang, Q.; Chen, F. A novel superhydrophobic hybrid nanocomposite material prepared by surface-initiated AGET ATRP and its anti-icing properties, J. Mater. Chem. A, 2 (2014) 9390-9399.

[23] Jiang, G.; Chen, L.; Zhang, S.; Huang, H. Superhydrophobic SiC/CNTs Coatings with Photothermal Deicing and Passive Anti-Icing Properties, ACS applied materials \& interfaces, 10 (2018) 36505-36511.

[24] Zhan, Y.; Ruan, M.; Li, W.; Li, H.; Hu, L.; Ma, F.; Yu, Z.; Feng, W. Fabrication of anisotropic PTFE superhydrophobic surfaces using laser microprocessing and their self-cleaning and anti-icing behavior, Colloids and Surfaces A: Physicochemical and Engineering Aspects, 535 (2017) 8-15.

[25] Ruan, M.; Zhan, Y.; Wu, Y.; Wang, X.; Li, W.; Chen, Y.; Wei, M.; Wang, X.; Deng, X. Preparation of PTFE/PDMS superhydrophobic coating and its anti-icing performance[J]. RSC Adv. 2017, 7(66):41339-41344. 
[26] Shen, Y.; Tao, J.; Tao, H.; Chen, S.; Pan, L.; Wang, T. Anti-icing potential of superhydrophobic Ti6A14V surfaces: ice nucleation and growth, Langmuir : the ACS journal of surfaces and colloids, 31 (2015) 10799-10806.

[27] Lei, S.; Wang, F.; Fang, X.; Ou, J.; Li, W. Icing behavior of water droplets impinging on cold superhydrophobic surface, Surface and Coatings Technology, 363 (2019) 362-368.

[28] Wu, X.; Tang, Y.; Silberschmidt, V. V.; Wilson, P.; Chen, Z. Mechanically Robust Transparent Anti-Icing Coatings: Roles of Dispersion Status of Titanate Nanotubes, Advanced Materials Interfaces, 5 (2018) 1800773.

[29] Li, C.; Li, X.; Tao, C.; Ren, L.; Zhao, Y.; Bai, S.; Yuan, X. Amphiphilic Antifogging/Anti-Icing Coatings Containing POSS-PDMAEMA-b-PSBMA, ACS applied materials \& interfaces, 9 (2017) 22959-22969.

[30] Du, P.; Wen, J.; Zhang, Z. Z.; Song, D.; Ouahsine, A.; Hu, H. B. Maintenance of air layer and drag reduction on superhydrophobic surface, Ocean Engineering, 130 (2017) 328-335. 
Table 1. Properties of hydrophobic surface based on various structure

Scheme 1. Schematic illustration of the mechanism for good anti-icing performance of superhydrophobic surface based on (A) assemblies of nanoparticles and (B) polymer nanocomposites. (C)Optical photographs of (a) pure glass and superhyhydrophobic surface coated glass with various WACs of (b) $150.7^{\circ}$ and (c) $173.1^{\circ}$ immersed in water.

Fig.1.(A)XRD and (B)FT-IR spectra of $\mathrm{SiO}_{2}$ nanoparticles before and after modification with OTS. (C)SEM image, (D)TEM image and (E)EDS spectrum of $\mathrm{SiO}_{2}$ nanoparticles modified with OTS.

Fig.2.The SEM images of (A) pure EP surface and surface prepared by various concentrations of nanoparticle dispersion solution, (B) $2.0 \mathrm{wt} \%$ and (C) $5.0 \mathrm{wt} \%$. The cross section SEM images of nanoparticle/EP coating prepared by various concentrations of nanoparticle dispersion solution, (D) $2.0 \mathrm{wt} \%$ and (E) $5.0 \mathrm{wt} \%$. The 3D surface morphology of superhydrophobic surface prepared by various concentrations of nanoparticle dispersion solution, (F) $2.0 \mathrm{wt} \%$ and $(\mathrm{G}) 5.0 \mathrm{wt} \%$. The insets of (A-C) are corresponding high-resolution SEM images. The insets of (E-G) are the WAC of pure EP surface, superhydrophobic surface $(2.0 \mathrm{wt} \%)$ and superhydrophobic surface $(5.0 \mathrm{wt} \%)$, respectively.

Fig.3.(A) Optical photographs and (B) adhensive strength of various surfaces coated Fe plate after pull-out tests. (a) pure EP, (b) $\mathrm{OTS}_{-} \mathrm{SiO}_{2} / \mathrm{EP}(2.0 \mathrm{wt} \%)$ and (c) $\mathrm{OTS}_{-} \mathrm{SiO}_{2} / \mathrm{EP}(5.0 \mathrm{wt} \%)$.

Fig.4.Optical photographs of water droplets on superhyhydrophobic surface with various WACs of (A) $173.1^{\circ}$ and (B) $150.7^{\circ}$ at an slope angle of $0^{\circ}$ and $5^{\circ}$.

Fig.5. Optical photographs of water droplets on superhyhydrophobic surface with WCA of $173.1^{\circ}$ at different cold temperatures of (A) $-20.0^{\circ} \mathrm{C}$ and (B) $-30.0^{\circ} \mathrm{C}$ at an slope angle of $0^{\circ}$, and at different slope angles of (C) $0.0^{\circ}$ and (D) $10.0^{\circ}$ at a cold temperature of $-40^{\circ} \mathrm{C}$. Optical photographs of water droplets on superhyhydrophobic surface with WCA of $150.7^{\circ}$ at different slope angles of (E) $0.0^{\circ}$ and (F) $10.0^{\circ}$ at a cold temperature of $-20.0^{\circ} \mathrm{C}$.

Fig.6. The icing process of water droplet on superhyhydrophobic surface with WCA of $173.1^{\circ}$ at different cold temperatures of $(\mathrm{A})-30.0^{\circ} \mathrm{C}$ and $(\mathrm{B})-40.0^{\circ} \mathrm{C}$. The icing process of water droplet on superhyhydrophobic surface with WCA of $150.7^{\circ}$ at different cold temperatures of (C) $-30.0^{\circ} \mathrm{C}$ and (D) $-40.0^{\circ} \mathrm{C}$. 
Fig.7. IR images of superhyhydrophobic surface with a WAC of $173.1^{\circ}$ at a cold temperature of (A) $-40.0^{\circ} \mathrm{C}$ and (B)- $30.0^{\circ} \mathrm{C}$, IR images of superhyhydrophobic surface with a WAC of $150.7^{\circ}$ at different cold temperatures of (C) $-40.0^{\circ} \mathrm{C}$ and (D) $-30.0^{\circ} \mathrm{C}$. (E)Temperature profiles of water droplets on superhyhydrophobic surface with various WACs and cold temperatures, (a) $173.1^{\circ}$ and $-40.0^{\circ} \mathrm{C}$, (b) $173.1^{\circ}$ and $-30.0^{\circ} \mathrm{C}$, (c) $150.7^{\circ}$ and $-40.0^{\circ} \mathrm{C},(\mathrm{d}) 150.7^{\circ}$ and $-30.0^{\circ} \mathrm{C}$.

Fig.8. (A) Schematic illustration of the sandpaper abrasion test. (B) WAC and SA of the superhydrophobic surface with a WCA of $173.1^{\circ}$ as function of abrasion cycle. (C) SEM image and (D) 3D surface morphology of superhydrophobic surface after 50 abrasion cycles. (E) Optical photographs of water droplets on superhydrophobic surface with a WCA of $173.1^{\circ}$ at an slope angle of $10.0^{\circ}$ and a cold temperature of $-40.0^{\circ} \mathrm{C}$ 
Table 1.

\begin{tabular}{|l|l|l|l|l|l|}
\hline Sample & $\begin{array}{l}\text { Contact } \\
\text { angle }\left({ }^{\circ}\right)\end{array}$ & $\begin{array}{l}\text { Sliding } \\
\text { angle }\left({ }^{\circ}\right)\end{array}$ & $\begin{array}{l}\text { Froze time } \\
(\mathrm{s})\end{array}$ & $\begin{array}{l}\text { Temperature } \\
\left({ }^{\circ} \mathrm{C}\right)\end{array}$ & References \\
\hline $\mathrm{SiO}_{2} / \mathrm{EP}$ & 173.1 & 0 & 372 & -40 & Present work \\
\cline { 3 - 6 } & & 562 & -30 & {$[11]$} \\
\hline $\mathrm{F}-\mathrm{SiO}_{2} / \mathrm{PDM}$ & 155.3 & 2 & 276 & -15 & {$[12]$} \\
\hline $\mathrm{ZnO} / \mathrm{PDMS}$ & 159.5 & 8 & $210 \pm 25$ & -15 & {$[13]$} \\
\hline $\mathrm{PPda} / \mathrm{SiO}_{2}$ & $167.0 \pm 2.0$ & 5 & 386 & -25 & {$[23]$} \\
\hline $\mathrm{SiC} / \mathrm{CNTs}$ & 161.0 & $<2$ & 66 & -30 & {$[24]$} \\
\hline $\mathrm{PTFE}$ & 168.4 & $\sim 3$ & 255 & -25 & {$[25]$} \\
\hline $\mathrm{PTFE} / \mathrm{PDMS}$ & 163.6 & $/$ & 120 & -6 & {$[26]$} \\
\hline $\begin{array}{l}\mathrm{Ti6Al} \\
\mathrm{S}-17\end{array}$ & 161.0 & 2 & 750 & -10 & {$[27]$} \\
\hline $\mathrm{SiO} / \mathrm{FA}$ & $165.5 \pm 2.7$ & $5 \pm 2$ & 80 & -12 & {$[28]$} \\
\hline $\begin{array}{l}\mathrm{Titanate} \\
\text { nanotube }\end{array}$ & 107.0 & $/$ & 349 & -15 & {$[29]$} \\
\hline $\mathrm{SIPN}$ & $105.8 \pm 0.4$ & $/$ & 334 & -15 & \\
\hline
\end{tabular}
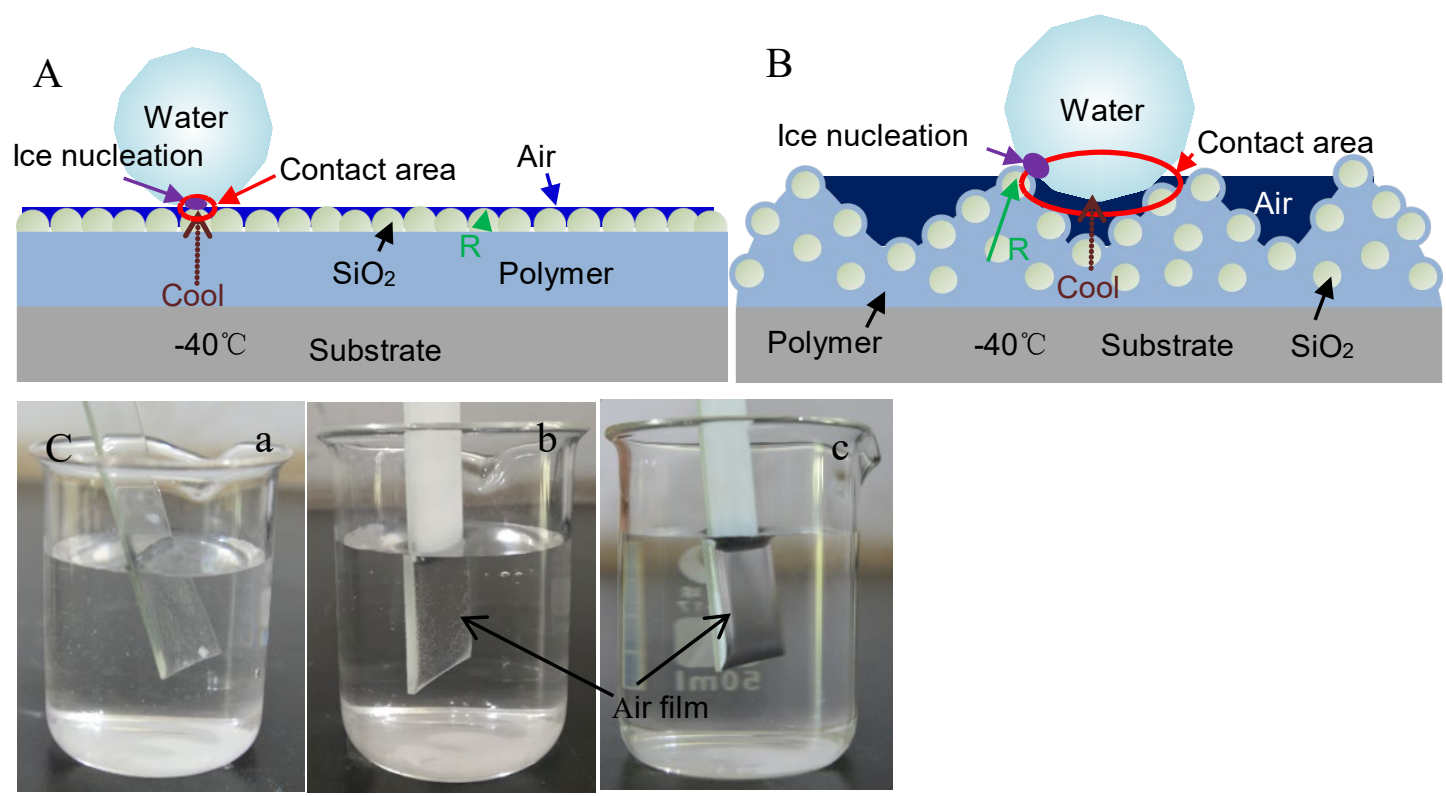

Scheme 1. 

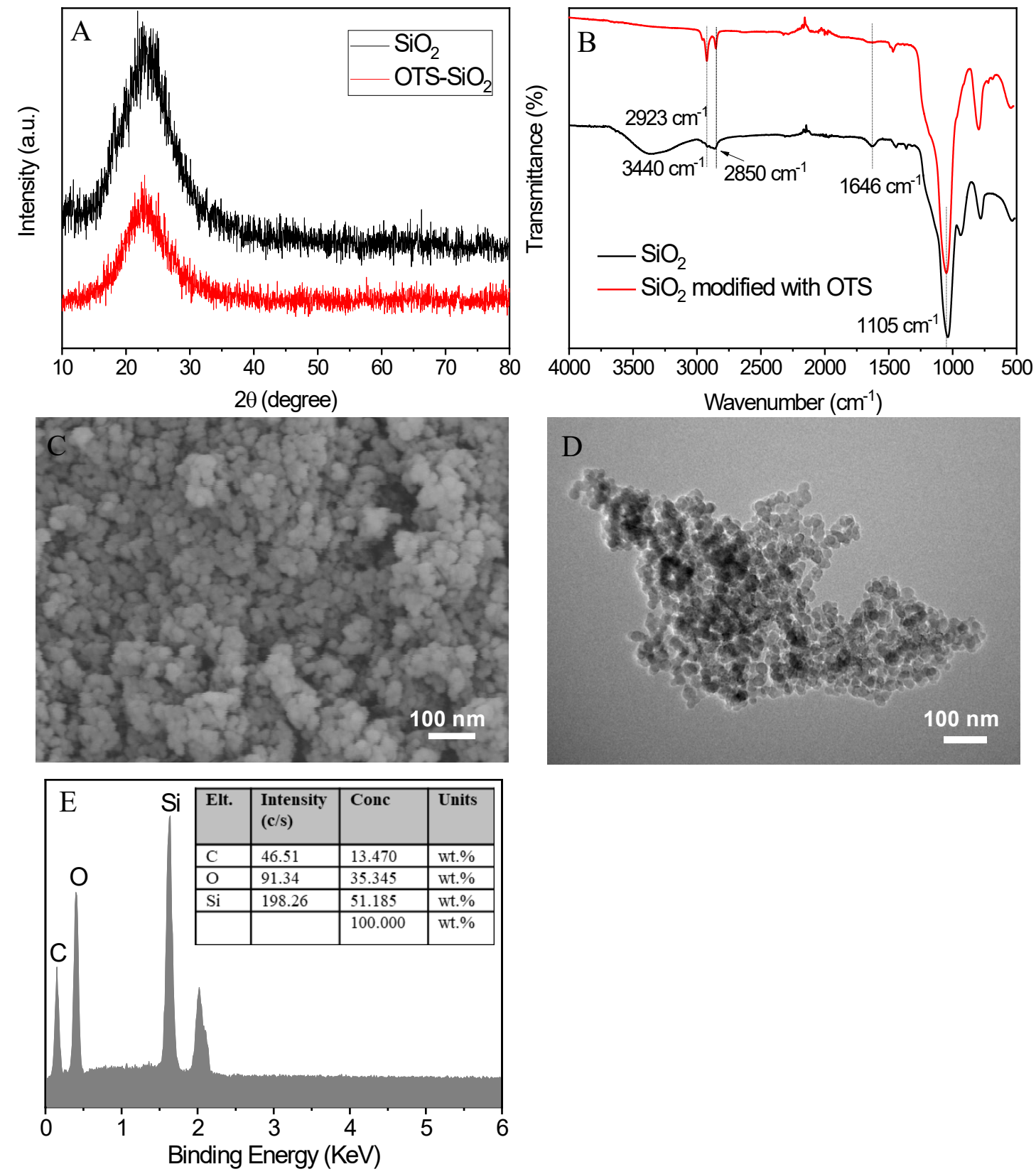

Fig.1. 

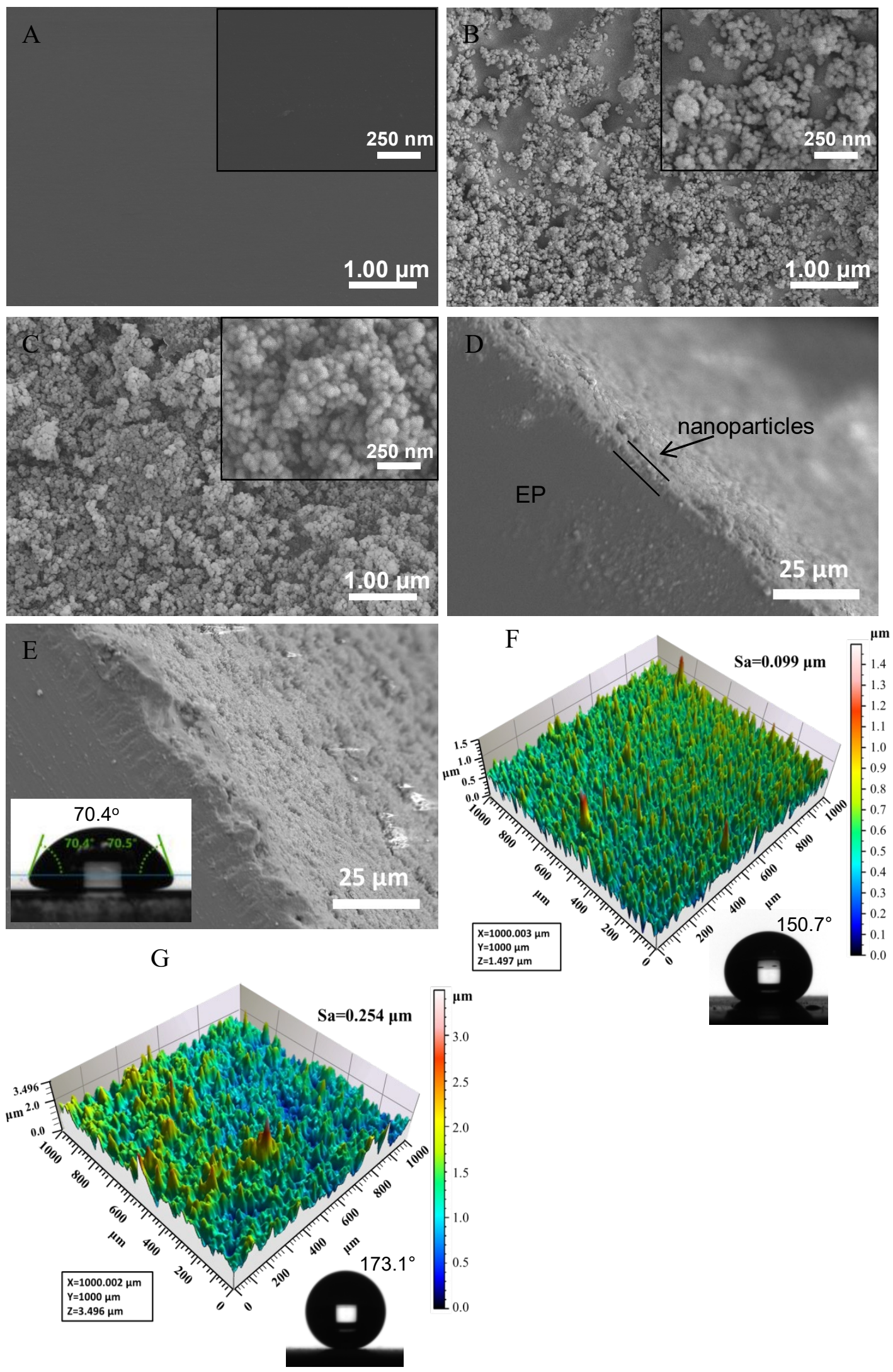

Fig.2. 

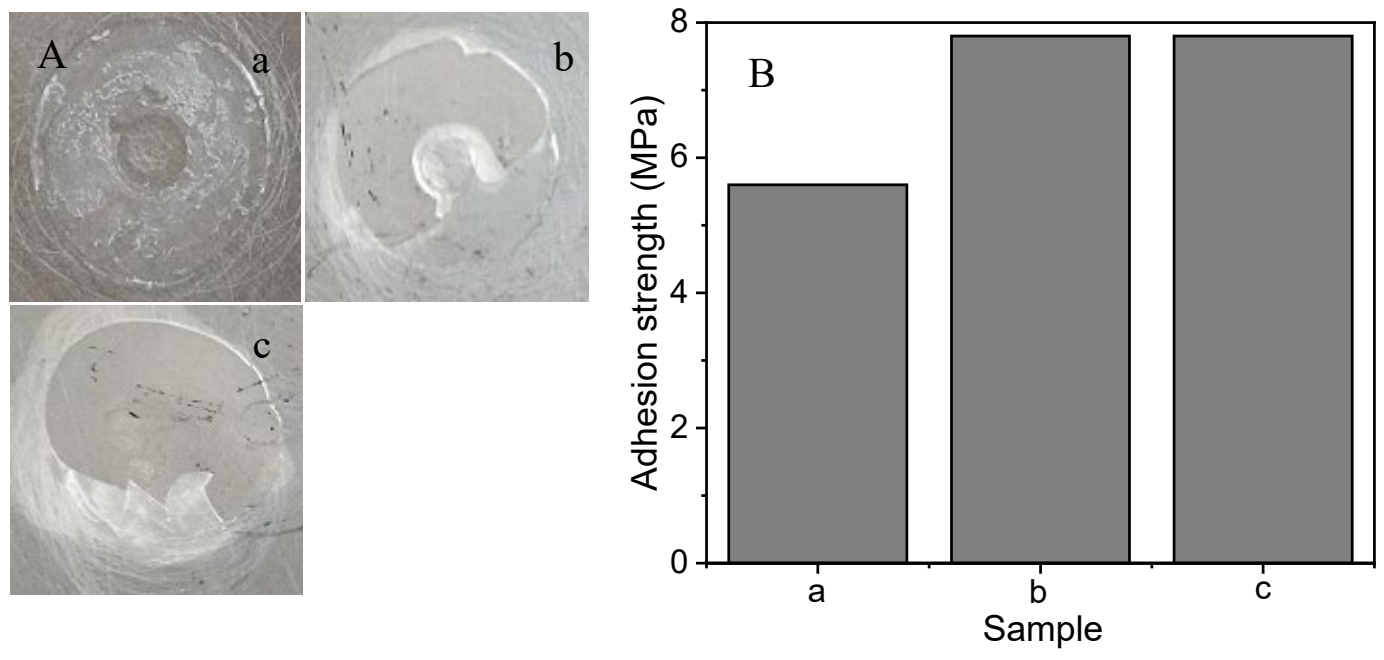

Fig.3. 

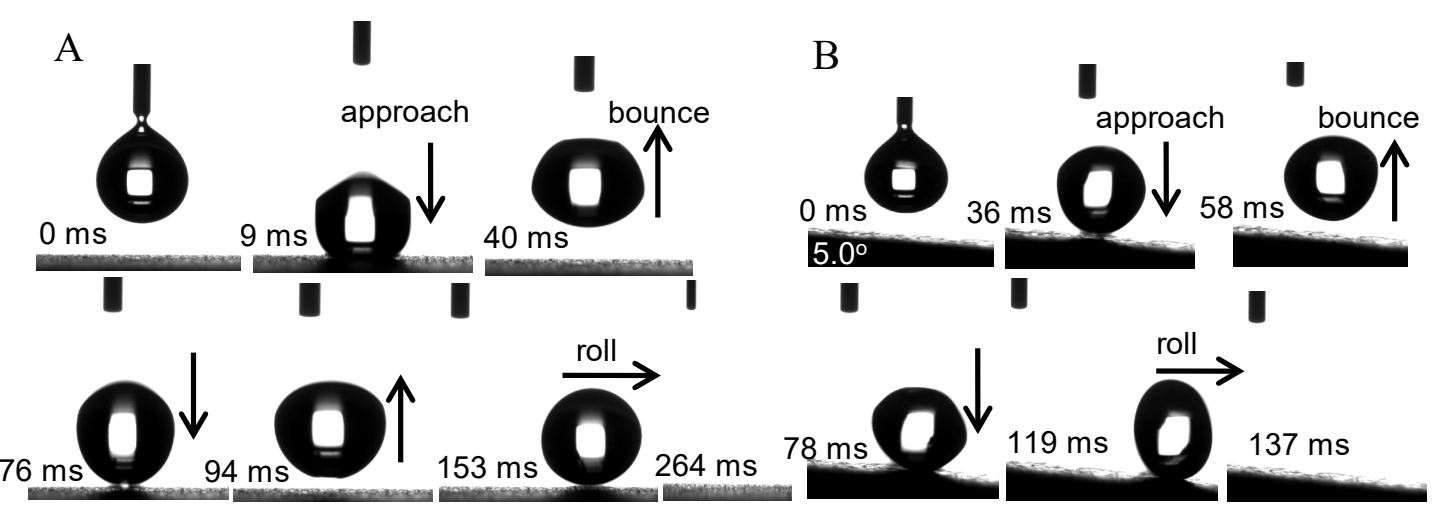

Fig.4. 


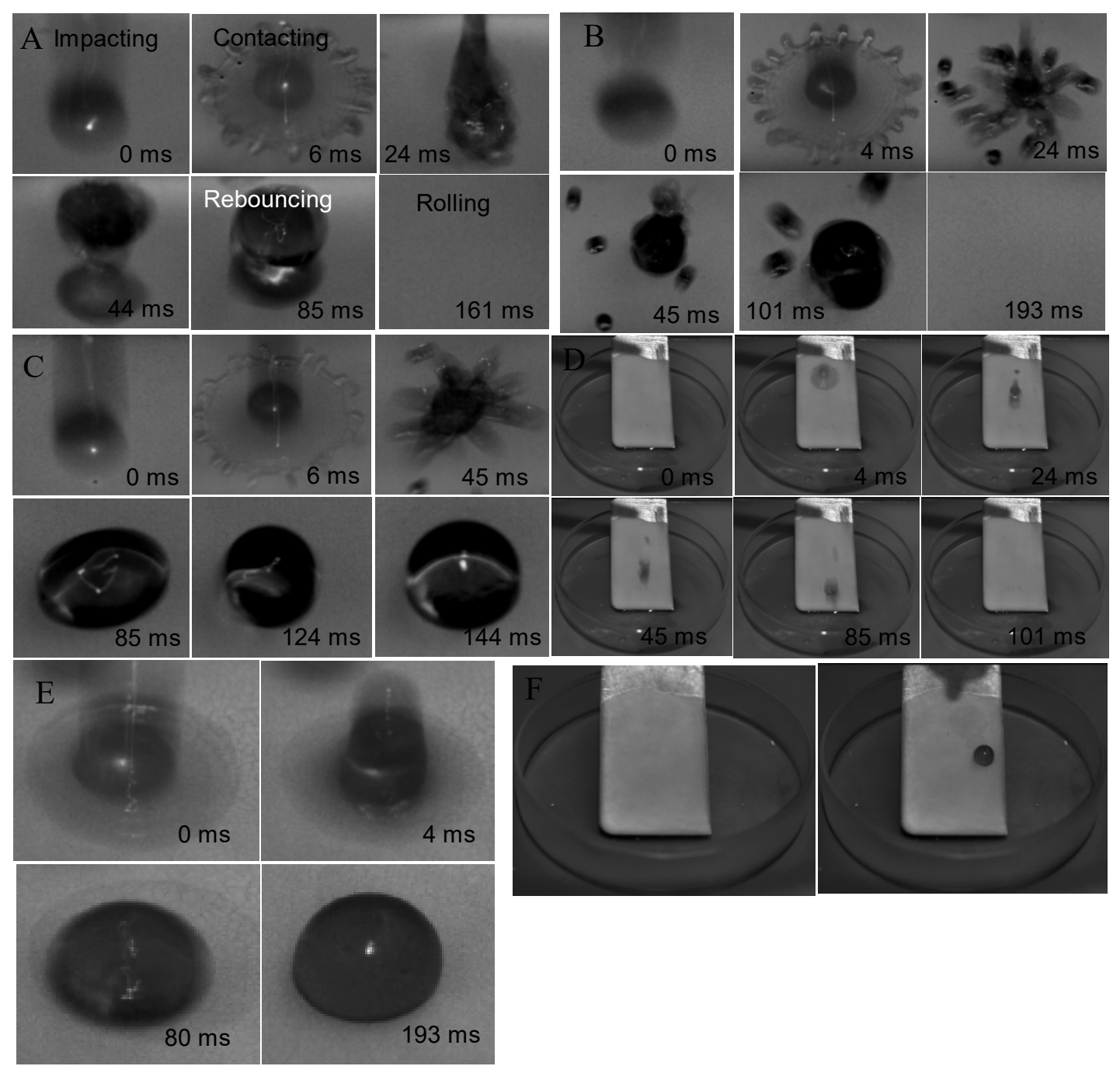

Fig.5. 


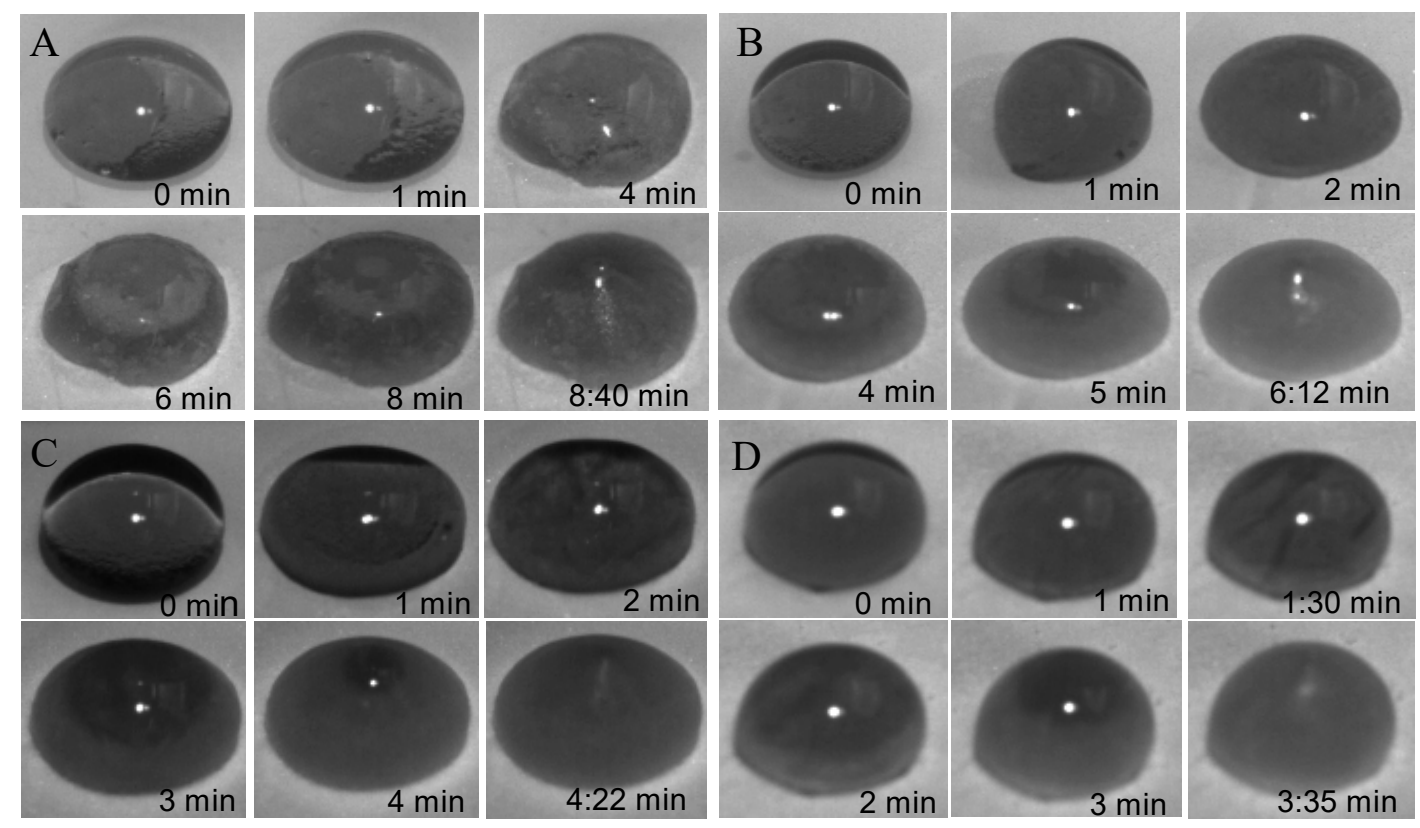

Fig.6. 

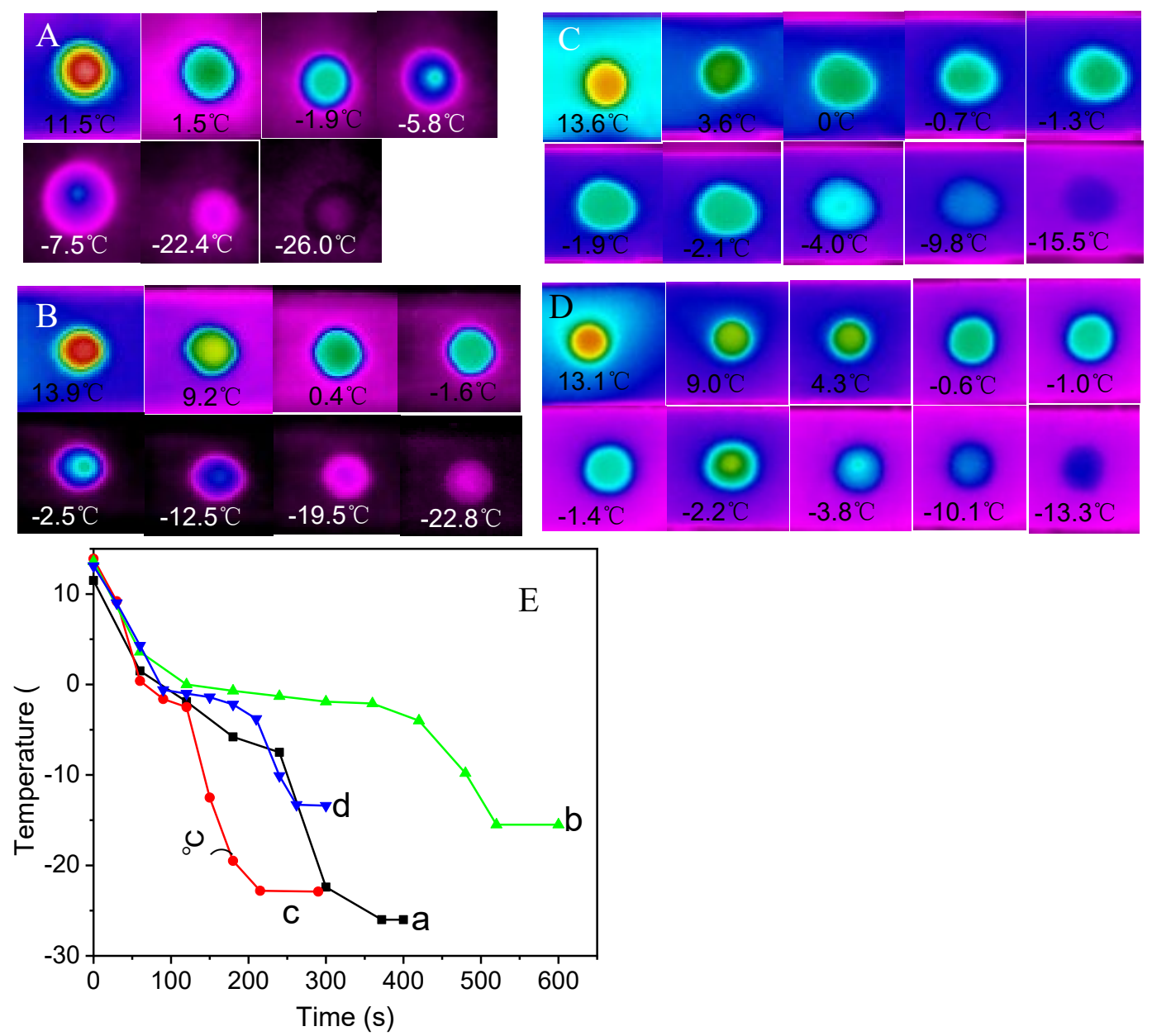

Fig.7. 

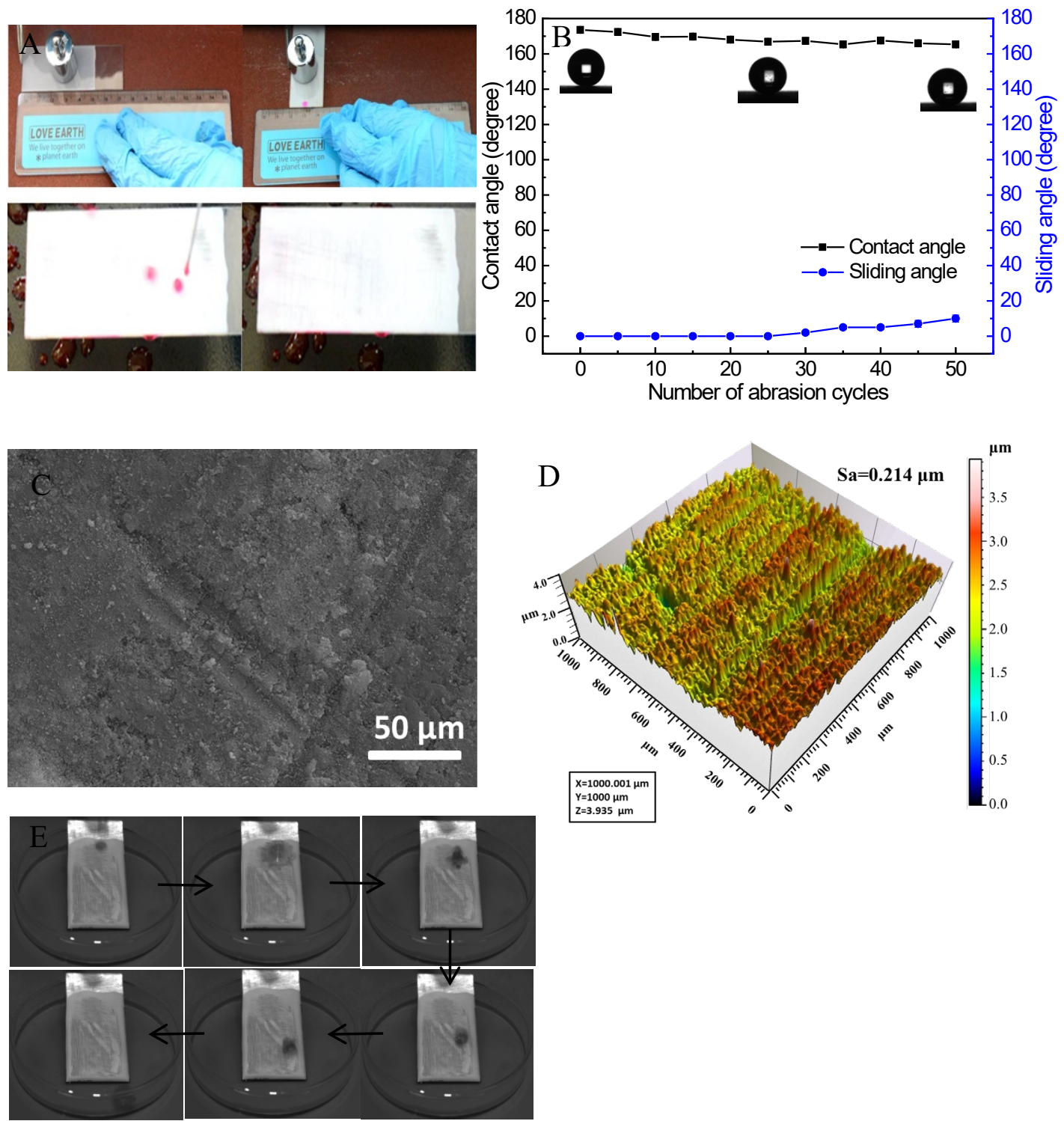

Fig.8. 